\title{
Photoemission and conduction currents in vacuum ultraviolet irradiated aluminum oxide
}

\author{
J. L. Lauer, J. L. Shohet, ${ }^{\text {a) }}$ C. Cismaru, ${ }^{\text {b) }}$ R. W. Hansen, ${ }^{\text {c) }}$ M. Y. Foo, and T. J. Henn \\ Center for Plasma-Aided Manufacturing and Department of Electrical \& Computer Engineering, \\ University of Wisconsin-Madison, Madison, Wisconsin 53706
}

(Received 6 July 2001; accepted for publication 25 October 2001)

\begin{abstract}
A temporary increase in the conductivity of aluminum oxide sputter deposited on the surface of aluminum wafers was made by exposure to vacuum ultraviolet (VUV) radiation produced by a synchrotron light source. The oxide was charged, either positively or negatively, by exposure to a nonreactive inductively coupled plasma, under typical plasma processing conditions. We show the dependence of the conductivity on the energy of the incoming radiation, and conclude that only those photons whose energy is above the band gap of the oxide are capable of producing a temporary increase in the conductivity. Two processes, photoemission and enhanced conductivity, create currents flowing across the oxide layer. A circuit model was developed to show the contributions from both processes to the total current. We conclude that VUV radiation may be used to significantly decrease plasma-induced surface charging of dielectrics. (C) 2002 American Institute of Physics. [DOI: 10.1063/1.1428790]
\end{abstract}

\section{INTRODUCTION}

During plasma processing, charging of dielectrics plays a leading role within the damage mechanisms of semiconductor devices and plasma-processed materials in general. This damage mechanism is greatly influenced by plasmaemitted x-ray, vacuum ultraviolet (VUV), and ultraviolet radiation. ${ }^{1-3}$ It was determined that most processing plasmas emit radiation in the VUV energy band of 4-30 eV, with most of the radiation above $9 \mathrm{eV}$, the latter of which is approximately the energy band gap of $\mathrm{SiO}_{2},{ }^{4}$ and more than the energy band gap of other dielectrics used in semiconductor device manufacturing (e.g., $6 \mathrm{eV}$ for $\mathrm{Si}_{x} \mathrm{~N}_{y}$ and $8.3 \mathrm{eV}$ for $\mathrm{Al}_{2} \mathrm{O}_{3}$ ). The radiation is absorbed in the exposed oxide layers and it results in the generation of electron-hole pairs. Although it has been established that electron-hole-pair generation in the oxide increases the $\mathrm{SiO}_{2}$ bulk and interface trapped-charge density, which may affect device reliability, ${ }^{5,6}$ plasma VUV irradiation of oxides can have a beneficial effect by inducing a temporarily enhanced oxide conductivity. This can reduce dielectric charging, especially that induced by electron-shading effects ${ }^{7}$ during plasma etching of high aspect-ratio devices, by providing a safe way to discharge these structures and, thus, minimize charging damage. The enhanced conductivity can also have beneficial effects on the etching properties of oxides such as reduction of notching, sidewall bowing, and trenching. In opposition to this effect, VUV photons incident on the surface of the dielectric may concomitantly cause photoemission of electrons and/or polarization of the dielectric, ${ }^{8}$ resulting in a net positive charge on the surface.

\footnotetext{
a) Author to whom correspondence should be addressed; electronic mail: shohet@engr.wisc.edu

b) Present address: Conexant Systems, Inc., Newburg Park, CA 91320.

c) Also with: Synchrotron Radiation Center, University of WisconsinMadison, Madison, WI 53706.
}

It has been previously shown, by exposure to synchrotron radiation in the above energy range, that a temporary increase in oxide surface conductivity was produced while the oxide was exposed to VUV radiation. ${ }^{9}$ The purpose of this work is to show the energy dependence of the surface conductivity in $\mathrm{Al}_{2} \mathrm{O}_{3}$ and that a net current is drawn through the oxide-caused by a combination of the photoemission/ polarization and the temporarily enhanced conductivity. By suitably biasing the substrate, we can decrease the photoemission current and retain the conduction current, thus permitting depletion of the previously stored charge in and around the VUV exposed region.

First, we demonstrate the dependence of the $\mathrm{Al}_{2} \mathrm{O}_{3}$ surface conductivity on the energy of the incoming radiation and we show that the peak conductivity occurs for irradiation with photons with an energy of approximately $18 \mathrm{eV}$. Second, by exposing precharged oxide samples to VUV, we show that the regions exposed to VUV radiation exhibit charge depletion in and around the exposed areas. Finally, we measure the current drawn by the oxide-coated wafer as a function of time for various biasing arrangements under VUV exposure.

\section{EXPERIMENTAL ARRANGEMENTS}

It was desired that the investigation of the VUV-induced conductivity on an oxide-coated wafer, as a function of the energy of the plasma VUV radiation, take place under exposure of the test structures to processing plasmas, while the VUV-emission intensity and wavelength is measured. However, these measurements are not possible during plasma exposure because the plasma provides an additional current path that interferes with our measurements. To eliminate this, we used monochromatic synchrotron radiation in the same photon energy range to expose the test structures under vacuum. The advantage of using monochromatic synchrotron 


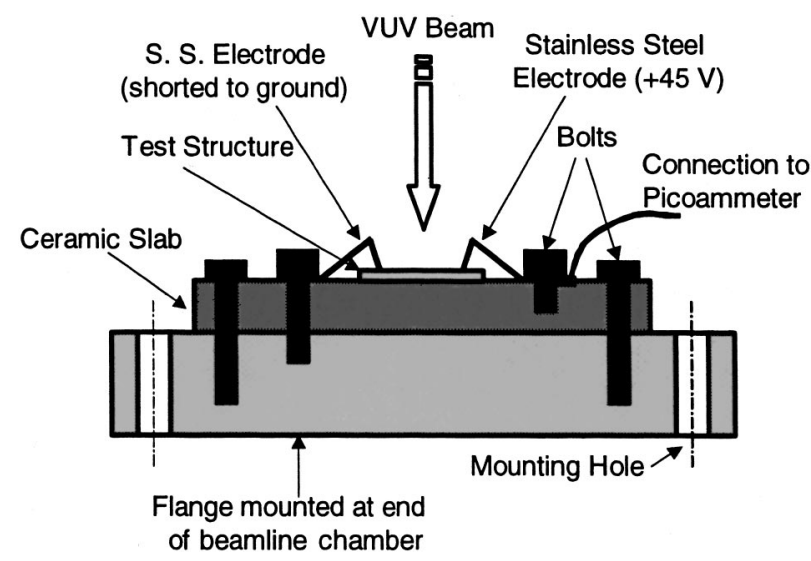

FIG. 1. Experimental setup used for the measurement of oxide surface conductivity under illumination with monochromatic VUV light.

radiation is the ability to determine the dependence of measured quantities on the wavelength of the incoming radiation.

To achieve our first goal, we exposed oxide-coated wafers to synchrotron radiation at the Synchrotron Radiation Center, at the University of Wisconsin-Madison. The monochromatic light was supplied by the Aladdin synchrotron storage ring, passing through a VUV monochromator. The electron beam used to generate the synchrotron radiation had a current of up to $250 \mathrm{~mA}$, at an energy of $800 \mathrm{MeV}$. The VUV monochromator that was connected to the beamline is a normal-incidence Seya-Namioka monochromator, with an output energy range of $4-30 \mathrm{eV}$, and a bandpass of $3 \AA$.

The oxide-coated samples were mounted in a vacuum chamber coupled to the beamline monochromator. The mounting unit was positioned to the incident VUV beam normal to the surface of the wafer. The monochromatic synchrotron light was directed on the sample (the position of the light spot is shown in Fig. 1), with a spot dimension of $5 \mathrm{~mm} \times 15 \mathrm{~mm}$. During the measurements, the chamber was evacuated to pressures in the $10^{-8}$ Torr range. Two stainlesssteel electrodes were used to connect an outside currentvoltage circuit to the sample as also shown in Fig. 1.

The current across the surface of the oxide sample was measured with a bias voltage of $45 \mathrm{~V}$, as the synchrotron light energy was varied between 5 and $20 \mathrm{eV}$. The oxide, in this case $\mathrm{Al}_{2} \mathrm{O}_{3}$, has an energy gap of approximately $8.3 \mathrm{eV}$, which is very similar to that of $\mathrm{SiO}_{2}$. The oxide was deposited by reactive sputtering and is $3000 \AA$ thick. The dark current was measured by recording the currents while scanning the same energy range with the light shutter closed. This also assured that there were no light leaks or other coupling to the circuit. In all cases, the dark current was at least 1 order of magnitude less than the conduction current. In addition, making sure that the incoming radiation does not hit the electrodes eliminates photoemission currents from being induced in the circuitry. The net oxide surface current (measured current less dark current) was normalized to a constant electron beam current $(200 \mathrm{~mA})$ and the photon flux at 15 $\mathrm{eV}$. The normalized net steady-state current as a function of incident photon energy is shown in Fig. 2. Note that no significant increase in current occurs until the VUV photon energy approaches the band gap of the oxide. The current due

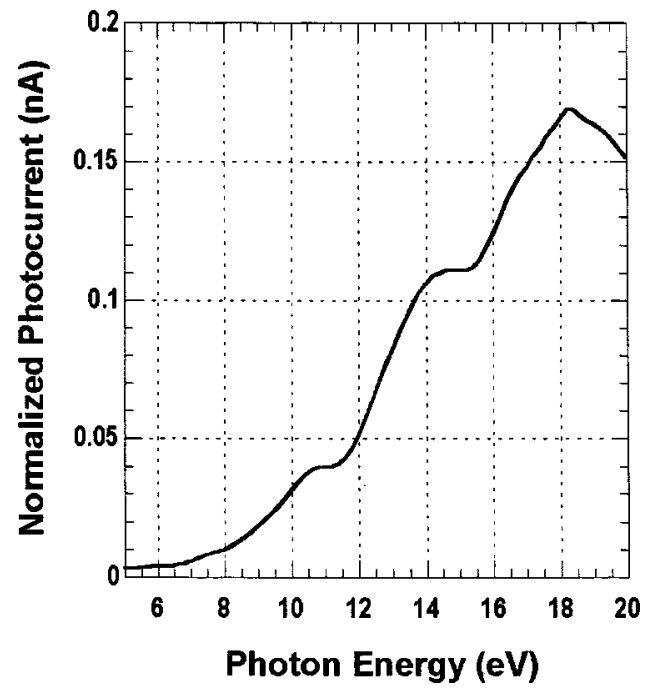

FIG. 2. Photon energy dependence of the normalized net steady state current along the $\mathrm{Al}_{2} \mathrm{O}_{3}$ surface.

to secondary emission of electrons by the stainless-steel electrodes is small compared to the current produced by electron-hole pair creation or photoemission in the oxide.

The effect of VUV radiation on depletion of charge is the key part of this work. In order to test this, samples were charged by placing them in a nonreactive argon inductively coupled plasma (ICP) for several minutes. The ICP power was $1 \mathrm{~kW}$ and two different sample bias conditions were used so as to produce a net positive or negative charge in the oxide. The former was achieved with $150 \mathrm{~W}$ rf bias power and the latter with zero bias power. Figure 3 shows a scan across the $11.5 \mathrm{~cm}$ sample using the contact potential difference (CPD) technique ${ }^{10}$ that was made before exposure to the VUV. The difference between positive and negative charging is quite clear.

The CPD technique employed in this work to determine the amount of charging induced by plasma exposure is an application of the Kelvin-probe technique, ${ }^{11}$ used for the

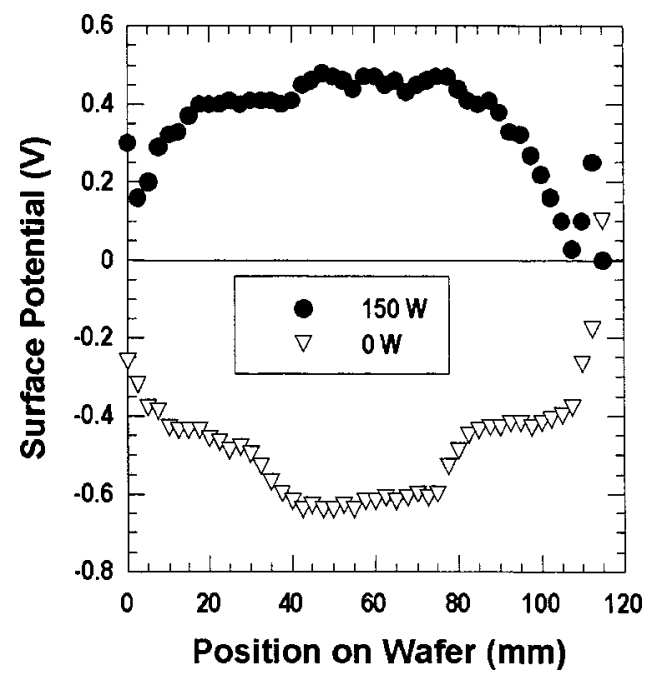

FIG. 3. CPD scans of the plasma-exposed samples show positive charge deposited for $150 \mathrm{~W}$ bias conditions and negative charge for no bias conditions. 


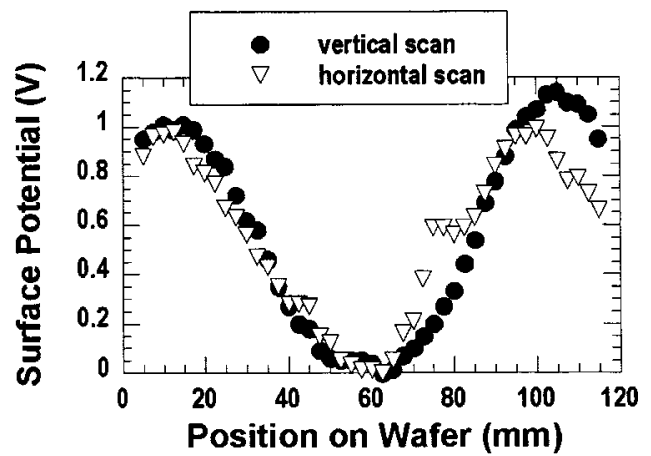

FIG. 4. Horizontal and vertical CPD scans of VUV exposed plasma-charged wafer.

measurement of work functions of different materials. The Kelvin-probe method measures the contact potential difference (difference in the work functions) between a vibrating Kelvin probe and the surface under analysis. In our case, by using oxide-coated wafers, the difference between measurements of plasma-exposed samples and nonexposed samples is the change in the oxide surface barrier, a direct function of the charge placed on the oxide's surface by plasma processing. Therefore, plasma induced oxide surface charge (density) can be expressed as $V_{\mathrm{CPD}} \varepsilon_{\mathrm{ox}} / d_{\mathrm{ox}}$, where $V_{\mathrm{CPD}}$ is the CPD potential measurement, $\varepsilon_{\mathrm{ox}}$ is the oxide permittivity, and $d_{\mathrm{ox}}$ is the oxide thickness.

\section{TRAPPED CHARGE MEASUREMENTS}

Since most plasma processing occurs with a positive charge being placed on the wafer, a CPD scan of a positively charged wafer was made after exposure to VUV, which is shown in Fig. 4 for zero bias conditions. Three VUV exposures were made on the sample at $15 \mathrm{eV}$ for 5,30 , and $300 \mathrm{~s}$. The first exposure was made at the exact center of the sample, while the remaining two were displaced above and below the center by about $10 \mathrm{~mm}$. Two CPD scans, at right angles to each other, show a dramatic change in the charge profiles. ${ }^{12}$ First, note that there is a significant decrease in charge at the center of the sample and an increase in charge toward the outside of the wafer. This is as a result of the VUV exposure that produces a temporary conductivity increase both on the surface of the oxide ${ }^{9}$ and in the bulk. ${ }^{13}$ The temporary increase in the conductivity in this area will tend to make this region an equipotential and charge flows outward from the central region to the outside, pushing other charges ahead of it and resulting in a pileup at the edge of the wafer. In order to achieve this condition, the electron-hole pairs produced by the VUV would have to be sufficiently dense so that ambipolar conditions exist, and thus the long VUV exposures described above were made. An explanation of the conductivity mechanism was discussed previously. ${ }^{13}$

When the VUV exposure is ended, the conductivity returns to its pre-exposure state, thus trapping the charge as is shown in Fig. 4. The symmetry in the scans is due to the fact that the first exposure was in the center of the wafer and the majority of the charge movement occurs during the first exposure. In addition, the other exposures were made only 10 $\mathrm{mm}$ above and below the center due to mechanical con-

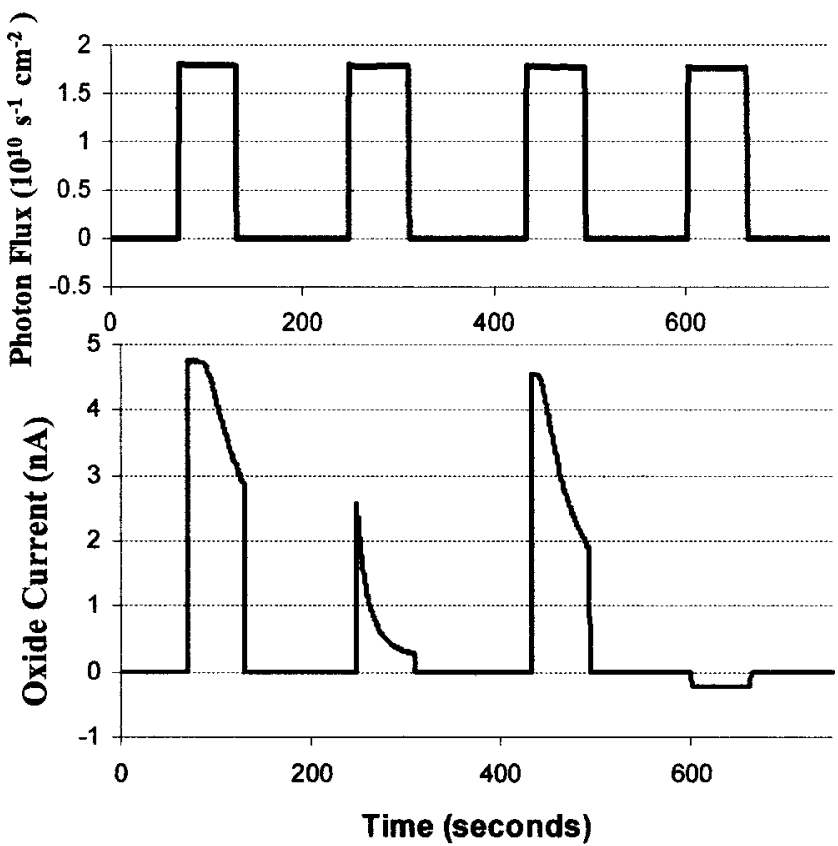

FIG. 5. Photon flux and current flowing out of the wafer under VUV exposure for four biasing arrangements: (left to right): $-90 \mathrm{~V}, 0 \mathrm{~V},-45 \mathrm{~V}$, and $+45 \mathrm{~V}$. The photon energy is $15 \mathrm{eV}$.

straints, a limited distance compared to the dimensions of the wafer. Subsequent exposures, although they can result in a temporary conductivity increase, are incident in a chargedepleted region, and thus do not significantly change the charge distribution. It is reasonable to conclude that, if a conducting path were placed on the edge of the VUV exposed region to ground, the charge would drain off as long as the VUV was turned on, thus providing a means to discharge the accumulated charge.

\section{CURRENT MEASUREMENTS}

Figure 5 shows the current to the substrate of the wafer as a function of time and the photon flux for several VUV exposures. It can be seen that a slow decay of the current takes place, when the wafer is negatively biased, but that the steady state value of the current is not zero. We conclude that the transient part of the current is due to photoemission, since eventually the oxide charges sufficiently positively to keep electrons from being emitted, and the remaining current combines both steady state photoemission and conduction through the oxide (see also Fig. 10). Note that the current immediately drops to zero when the VUV is turned off.

The net charge on the dielectric was then measured with the CPD method as shown in Fig. 6. The peaks of the charge correspond beam locations for each of the various current measurements in Fig. 5. This was accomplished by moving the wafer with respect to the photon beam. The lowest contact potential (lowest charge) occurs with the positive bias. Because the wafer was initially uncharged, the only charge that appears here is localized and is only that due to photoemission of the few electrons that escape the positive bias potential. 


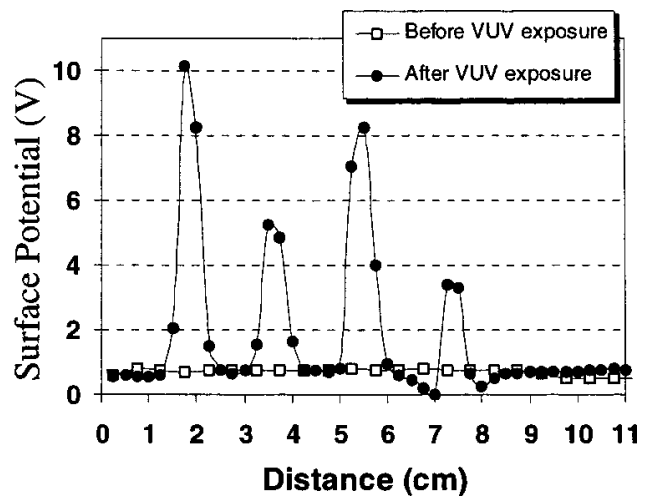

FIG. 6. CPD scan of the VUV exposed wafer in Fig. 5.

As an additional measurement, the substrate was floated with respect to the chamber, and its potential was measured before, during, and after VUV exposure. The potential rose to approximately $7 \mathrm{~V}$ during VUV exposure and remained constant until the VUV was turned off, when it decayed back toward zero. The $7 \mathrm{~V}$ potential is approximately the difference between the photon energy $(15 \mathrm{eV})$ and the band gap energy $(8.3 \mathrm{eV})$.

\section{ELECTRIC CIRCUIT MODEL}

In order to more fully understand the nature of the current measured during VUV exposure, a circuit model was developed. Figure 7 shows a drawing of the experimental arrangement.

As can be seen, incident VUV radiation results in photemission from the oxide layer. The photoemitted electrons have an initial energy, which we estimate to be on the order of $7 \mathrm{~V}$ and results in a photoemission current that we assume follows the Child-Langmuir law for a vacuum diode. In addition, a conduction current, $I_{\mathrm{ox}}$ flows through the oxide as shown. The substrate can be biased with an external dc supply, either positively or negatively. It should be emphasized that both currents cease when the VUV is turned off as can be seen in Fig. 5. In addition, Fig. 5 shows that reversing the substrate bias voltage effectively eliminates the photoemission current and no transient is observed, but conduction current still remains.

Figure 8 shows the resulting circuit diagram with the portions of the circuit that correspond to the aluminum oxide layer, the substrate, and the vacuum chamber identified. We

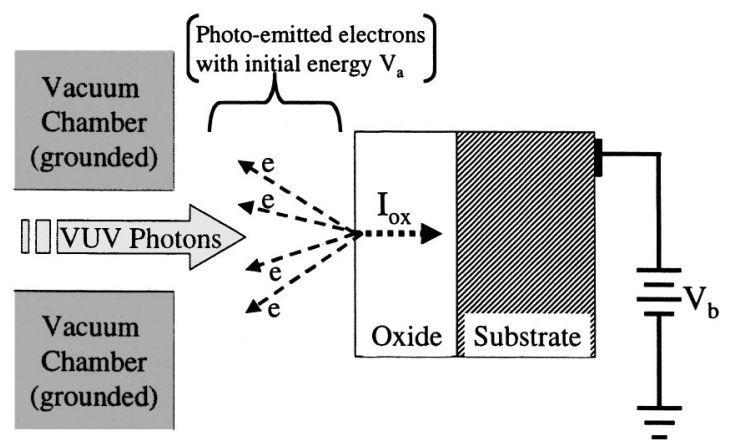

FIG. 7. Physical experimental setup.

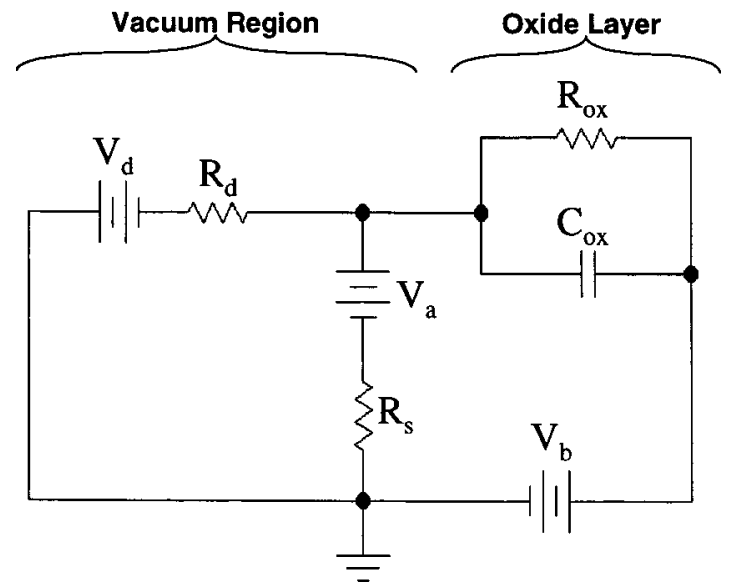

FIG. 8. Circuit model.

assume that the photoemission characteristic corresponds roughly to a piecewise-linear diode, whose voltage $V_{d}$ and resistance $R_{d}$ are fitting parameters. Additional fitting parameters are $V_{a}$, the initial energy of the photoemitted electrons, and $R_{S}$, the current limiting resistance of the VUV generator, which is inversely proportional to the VUV intensity. Both electrons and holes can contribute to the conduction current, but only electrons to the photoemission current. $R_{\mathrm{ox}}$ is the temporary resistance introduced by the VUV which is a function of the voltage across the oxide due to the FowlerNordheim tunneling effect. We model it as:

$$
R_{\text {ox }}=R_{0} \exp \left[-\xi\left(V-V_{b}\right)\right],
$$

where $R_{0}$ and $\xi$ are fitting parameters. $C_{\mathrm{ox}}$ is the effective capacitance across the oxide corresponding to the area of the VUV exposure. $V$ is the voltage on the oxide surface with respect to ground.

Since we do not know the division between the photoemission current and the conduction current, we find the Thevenin equivalent circuit for the diode and VUV circuit regions and then convert to a Norton equivalent circuit to find the oxide current. The circuit equation is:

$$
\frac{d V}{d t}+\frac{V}{C_{\mathrm{ox}}\left(R_{d}\left\|R_{s}\right\| R_{\mathrm{ox}}\right)}=-I_{0},
$$

where $V$ is the node voltage, $C_{\text {ox }}$ is the oxide capacitance, $R_{d}$ is the diode resistance, $R_{s}$ is the VUV source resistance, and $I_{0}$ is the Norton current source as shown below:

$$
I_{0}=\frac{V_{b}+\left[\left(\frac{V_{d}}{R_{d}}\right)+\left(\frac{V_{a}}{R_{s}}\right)\right]\left(R_{d} \| R_{s}\right)}{\left(R_{d} \| R_{s}\right)} .
$$

The symbol $\|$ means the parallel combination of the resistors listed. Equation (1) is solved numerically for the node voltage $V$. The oxide current is then the current passing through the parallel combination of $C_{\mathrm{ox}}$ and $R_{\mathrm{ox}}$ as shown in Fig. 9.

Assuming values for $V_{a}, V_{d}, C$, and $V_{b}$, we can then use the measured data to find algebraically $R_{o x}$ and the parallel combination of $R_{s}$ and $R_{d}$. We cannot separate these 


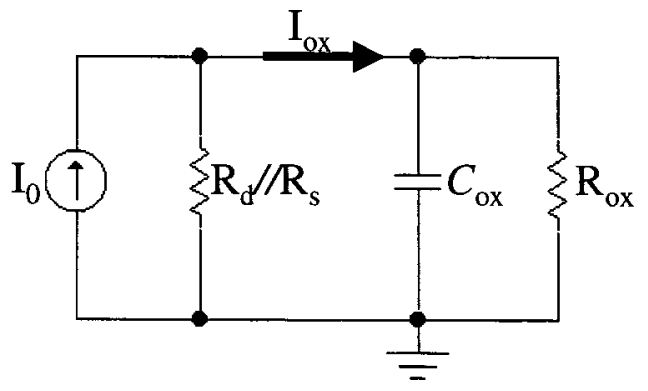

FIG. 9. Norton equivalent circuit model.

two resistors because there is no separate measurement of the photoemitted current. The values of the resistances we obtained are as shown in Table I.

Figure 10 shows the experimentally measured data with a plot of the computed current for a bias voltage of -90 . The agreement is quite good. We also can compute the effective conductivity of the oxide from the computed value of $R_{\text {ox }}$. We assume that the conducting region appears directly below the VUV beam, whose area is $5 \times 15 \mathrm{~mm}^{2}$. The $\mathrm{Al}_{2} \mathrm{O}_{3}$ oxide is $3000 \AA$ thick, and resulting bulk conductivity is: 1.48 $\times 10^{-16}(\Omega \mathrm{cm})^{-1}$.

\section{CONCLUSION}

In summary, we showed the dependence of the $\mathrm{Al}_{2} \mathrm{O}_{3}$ surface conductivity on the energy of the incoming radiation. We found the peak surface conductivity to occur for irradiation with photons with an energy of approximately $18 \mathrm{eV}$. Second, by exposing precharged oxide samples to VUV, we showed that the regions exposed to VUV radiation exhibit charge depletion in and around the exposed areas. The oxide was precharged, either positively or negatively, by exposure to nonreactive ICP, under typical plasma processing condi-

TABLE I. Fitting parameters of the electric circuit model.

\begin{tabular}{cl}
\hline \hline Measured or assumed quantity & \multicolumn{1}{c}{ Fit quantity } \\
\hline$C_{\mathrm{OX}}=4.2 \times 10^{-9} \mathrm{~F}$ & $R_{d} / / R_{s}=2.45 \times 10^{10} \Omega$ \\
$V_{a}=3 \mathrm{~V}$ & $\xi=0.012$ \\
$V_{d}=3 \mathrm{~V}$ & $R_{\mathrm{ox}}=2.7 \times 10^{11} \Omega$ \\
\hline
\end{tabular}

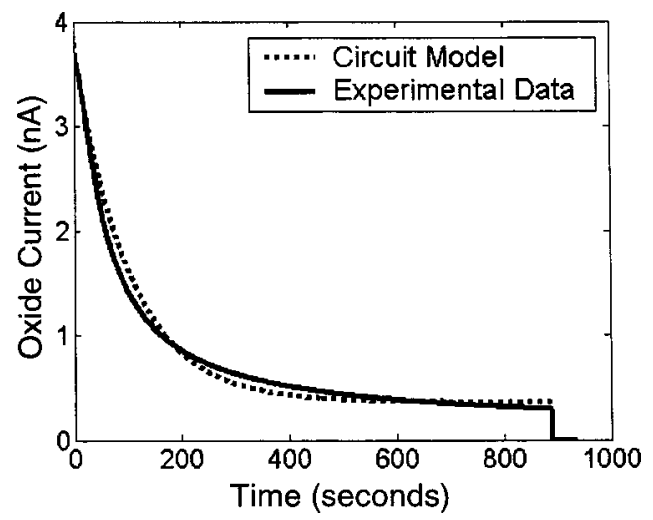

FIG. 10. Fit of computed current to experimental measurements for $V_{b}$ $=-90 \mathrm{~V}$.

tions. We conclude that VUV radiation can significantly decrease plasma-induced surface charging of dielectrics.

\section{ACKNOWLEDGMENTS}

This work was supported in part by Texas Instruments, Hewlett-Packard Company, LSI Logic, and the McNair Foundation. SRC is a national facility, funded by the National Science Foundation under Grant No. DMR-9531009.

${ }^{1}$ R. A. Gdula, IEEE Trans. Electron Devices 26, 644 (1979).

${ }^{2}$ T. Yunogami, T. Mizutani, K. Suzuki, and S. Nishimatsu, Jpn. J. Appl. Phys., Part 1 28, 2172 (1989).

${ }^{3}$ T. Mizutani, in International Symposium on Plasma Process-Induced Damage, edited by K. P. Cheung, M. Nakamura, and C. T. Gabriel (NCCAVS, Sunnyvale, CA, 1996), p. 157.

${ }^{4}$ C. Cismaru and J. L. Shohet, Appl. Phys. Lett. 74, 2599 (1999).

${ }^{5}$ S. A. Bell and D. W. Hess, J. Electrochem. Soc. 139, 2904 (1992).

${ }^{6}$ A. W. Flounders, S. A. Bell, and D. W. Hess, J. Electrochem. Soc. 140, 1414 (1993).

${ }^{7}$ K. Hashimoto, Jpn. J. Appl. Phys., Part 1 32, 6109 (1993)

${ }^{8}$ R. A. Moreno and S. Mascarenhas, Proceedings of the 2nd International Conference on Electrets, Charge Storage and Transport in Dielectrics, The Electrochemical Society, Princeton, NJ, 1973, p. 348.

${ }^{9}$ C. Cismaru, J. L. Shohet, and J. P. McVittie, Appl. Phys. Lett. 76, 2191 (2000).

${ }^{10}$ P. Edelman, A. M. Hoff, L. Jastrzebski, and J. Lagowski, Proc. SPIE 2337, 154 (1994).

${ }^{11}$ W. A. Zisman, Rev. Sci. Instrum. 3, 367 (1932).

${ }^{12}$ C. Cismaru, J. L. Shohet, J. L. Lauer, R. W. Hansen, and S. Ostapenko, Appl. Phys. Lett. 77, 3914 (2000).

${ }^{13}$ C. Cismaru and J. L. Shohet, J. Appl. Phys. 88, 1742 (2000). 